Article

\title{
An Improved Method for Road Extraction from High-Resolution Remote-Sensing Images that Enhances Boundary Information
}

\author{
Shuai Wang ${ }^{1}{ }^{\oplus}$, Hui Yang ${ }^{2}$, Qiangqiang $\mathrm{Wu}^{3}{ }^{3}$, Zhiteng Zheng ${ }^{3, *}$, Yanlan $\mathrm{Wu}^{3,4}$ and Junli $\mathrm{Li}^{5}$ \\ 1 School of Resource and Environmental Science, Wuhan University, Wuhan 430079, China; \\ 2010282050194@whu.edu.cn \\ 2 Institutes of Physical Science and Information Technology, Anhui University, Hefei 230601, China; \\ yanghui@ahu.edu.cn \\ 3 School of Resources and Environmental Engineering, Anhui University, Hefei 230601, China; \\ x18301096@stu.ahu.edu.cn (Q.W.); wuyanlan@ahu.edu.cn (Y.W.) \\ 4 Anhui Engineering Research Center for Geographical Information Intelligent Technology, \\ Hefei 230601, China \\ 5 School of Resources and Environment, Anhui Agricultural University, Hefei 230036, China; \\ lijunli866@ahau.edu.cn \\ * Correspondence: x17201009@stu.ahu.edu.cn
}

Received: 7 January 2020; Accepted: 5 April 2020; Published: 7 April 2020

\begin{abstract}
At present, deep-learning methods have been widely used in road extraction from remote-sensing images and have effectively improved the accuracy of road extraction. However, these methods are still affected by the loss of spatial features and the lack of global context information. To solve these problems, we propose a new network for road extraction, the coord-dense-global (CDG) model, built on three parts: a coordconv module by putting coordinate information into feature maps aimed at reducing the loss of spatial information and strengthening road boundaries, an improved dense convolutional network (DenseNet) that could make full use of multiple features through own dense blocks, and a global attention module designed to highlight high-level information and improve category classification by using pooling operation to introduce global information. When tested on a complex road dataset from Massachusetts, USA, CDG achieved clearly superior performance to contemporary networks such as DeepLabV3+, U-net, and D-LinkNet. For example, its mean IoU (intersection of the prediction and ground truth regions over their union) and mean $F 1$ score (evaluation metric for the harmonic mean of the precision and recall metrics) were $61.90 \%$ and $76.10 \%$, respectively, which were $1.19 \%$ and $0.95 \%$ higher than the results of D-LinkNet (the winner of a road-extraction contest). In addition, CDG was also superior to the other three models in solving the problem of tree occlusion. Finally, in universality research with the Gaofen-2 satellite dataset, the CDG model also performed well at extracting the road network in the test maps of Hefei and Tianjin, China.
\end{abstract}

Keywords: road extraction; deep learning; coordconv; global attention; high resolution

\section{Introduction}

The establishment of road databases is of great significance to the development of modern cities. At present, the extraction of road networks from high-resolution remote-sensing images has been widely used in urban services such as urban planning [1], vehicle navigation [2] and geographic information management $[3,4]$. However, due to the macro-scale nature of remote-sensing images, the 
complexity of road networks and the interference of other objects (such as parking lots, building roofs and trees), it is difficult to extract roads effectively [5,6].

At present, the methods for remote-sensing artificial road information extraction mainly include pixel-based [7-12], object-oriented [13-18], and deep learning methods [19-27]. Pixel-based methods mainly extract roads by analyzing the spectral information of the pixels, such as road extraction methods based on mean shift [7], adaptive domain [8], threshold filtering and line segment matching rules [12]. Pixel-based road extraction methods mainly use the spectrum of the image, which has a certain effect on simple road network extraction. However, these methods lack information such as the spatial context of the features and the texture structure, which has a "salt and pepper" effect.

In the object-oriented extraction method, the road is taken as an object and the information model is built to extract the road from the remote-sensing images. This method has good noise immunity and applicability. Compared with the pixel method, the accuracy is also improved. For example, Das et al. [13] used the local linear features of multispectral road images combined with the support vector machine model to achieve road extraction. However, Cheng et al. [14] made good use of the texture and geometric features of remote-sensing images in road extraction of rural and suburban areas. In work on urban road extraction, Senthilnath et al. [15], after preprocessing a remote-sensing image, used the method of texture progressive analysis and a normalized cut algorithm to realize the automatic detection of urban roads based on the road structure, spectral information and geometric features. Xin et al. [17] also proposed a road extraction method based on multiscale structural features and a support vector machine and successfully extracted the centerline of the road on two multispectral datasets. Although the extraction performance of such methods has been improved, it is easy to misclassify pixels that are adjacent spatially and have similar structural features. The design of the classification rules is very complicated, and the accuracy of extraction also needs to be improved.

Deep learning methods have been increasingly applied to information extraction from high-resolution satellite images due to their good performance and generalization ability [28,29]. Since Mihi et al. [30] applied deep learning methods to road extraction, other deep learning models have been applied to road extraction research [5,19-27]. Because fully convolutional networks (FCN) has achieved good results in semantic segmentation, many excellent FCN-based networks, such as U-net [31], SegNet [32], and DeepLabV3+ [33], have achieved the advanced performance in image segmentation and have been widely used for road extraction. For example, Wei et al. [19] proposed a road extraction model based on the road structure refined convolutional neural network (RSRCNN) and constructed a new loss function that improved road extraction accuracy. Zhang et al. [5] also proposed a new road extraction method based on U-net, which improved the model's semantic segmentation ability, reduced the loss of information, and effectively improved the accuracy of road extraction. To reduce misclassification in road classification tasks, Panboonyuen et al. [22] proposed an improved deep convolutional neural network (DCNN) framework that introduced the three mechanisms of landscape metrics (LMs), conditional random fields (CRFs), and exponential linear unit (ELU) in the network, which improved the extraction accuracy of the road and reduced misclassification. However, FCN mainly expands the receptive field and obtains context information through continuous pooling layers, which will cause the loss of small targets and important spatial details during the downsampling process. To overcome the loss of spatial information and obtain more global context information, in the 2018 CVPR (Computer Vision and Pattern Recognition) DeepGlobe Road Extraction Challenge, Zhou et al. [23] used dilated convolution to expand the receptive field and save a certain amount of spatial information while acquiring highly discriminative feature information, which improved the accuracy of the extraction and ultimately achieved excellent results in the competition. However, continuous dilated convolution will still cause the loss of spatial information, produce a "chessboard effect" and consume considerable computer memory.

Then, to reduce the loss of spatial information at multiple levels, Liu et al. [34] proposed an important coordinate convolution module. Coordinate convolution adds two channels to the original convolution to store horizontal and vertical pixel information to obtain spatial information. 
Yao et al. [35] applied coordinate convolution to land-use classification, effectively strengthened the edge information, and improved the accuracy of classification.

Therefore, to reduce the loss of spatial information at multiple levels, strengthen the global context of FCNs and improve the accuracy of road extraction, in this paper we design a novel encoder-decoder network called the coord-dense-global network to extract roads from remote sensing imagery and achieve better performance. DenseNet uses dense connectivity to connect multilevel feature maps and is considered a powerful feature extractor that can effectively reduce information loss [36]; this is very suitable for extending FCN networks for semantic segmentation [37]. The coord-dense-global network mainly use DenseNets to extract multilevel feature maps. The coordconv module is used to strengthen boundary information and fine details. At the same time, the global attention module is also introduced to highlight high-level features in the encoder part, which can maintain the continuity of roads.

The remainder of this paper is organized as follows: Section 2 presents the proposed methods. Section 3 shows the experimental details and results. Section 4 presents the comparison and analysis of the proposed model, and the conclusions are presented in Section 5.

\section{Materials and Methods}

\subsection{Proposed Network Architecture}

In general, convolutional networks are composed of a series of connected convolutional layers in which $L$ layers will produce $L$ connections. There exists a nonlinear transformation function $F_{L}$ in each layer, which usually contains convolutions, rectified linear units [38], and pooling. If the input and output of layer $L$ are $X_{L-1}$ and $X_{L}$, respectively, the layer transition can be defined as:

$$
X_{L}=F_{L}\left(X_{L-1}\right)
$$

This simple layer transition will lead to information loss and weaken the information flow between the layers. DenseNet has been widely used to solve such problems in image segmentation [39], as its dense connectivity allows the reuse of information from previous layers and reduces the number of parameters, making the network more easily trainable. In our dense connectivity module, the feature maps of all preceding layers are connected to subsequent layers, so layer $L$ receives the feature maps from all previous layers $\left(X_{0}, X_{1}, X_{2}, \ldots, X_{L-1}\right)$ as input. The layer transformation can thus be described as:

$$
X_{L}=F_{L}\left(\left[X_{0}, X_{1}, X_{2}, \cdots, X_{L-1}\right]\right)
$$

where $\left[X_{0}, X_{1}, X_{2}, \ldots, X_{L-1}\right]$ is the concatenation of all feature maps from all preceding layers. The non-linear transformation function $F_{L}$ often consists of three consecutive parts: a batch normalization layer [40], a layer of rectified linear units, and a convolutional layer. In addition, DenseNet is designed to have a growth rate that suppresses the redundancy of feature maps and improves the efficiency of the network.

Due to the efficiency of dense connectivity, we used an encoder-decoder architecture based on the fully convolutional DenseNet (Figure 1). Because roads are thin and long, we used the coordconv layer to strengthen informational detail and reduce the loss of spatial features in the network's top layer [35]. In the encoder, a series of dense blocks were applied to extract abstract features, with two global attention layers at the bottom. In the decoder, the high-level features after upsampling were directly concatenated with low-level features from the encoder using skip layers to form a new dense block input. Finally, the binary segmentation map was output following a convolutional layer. 

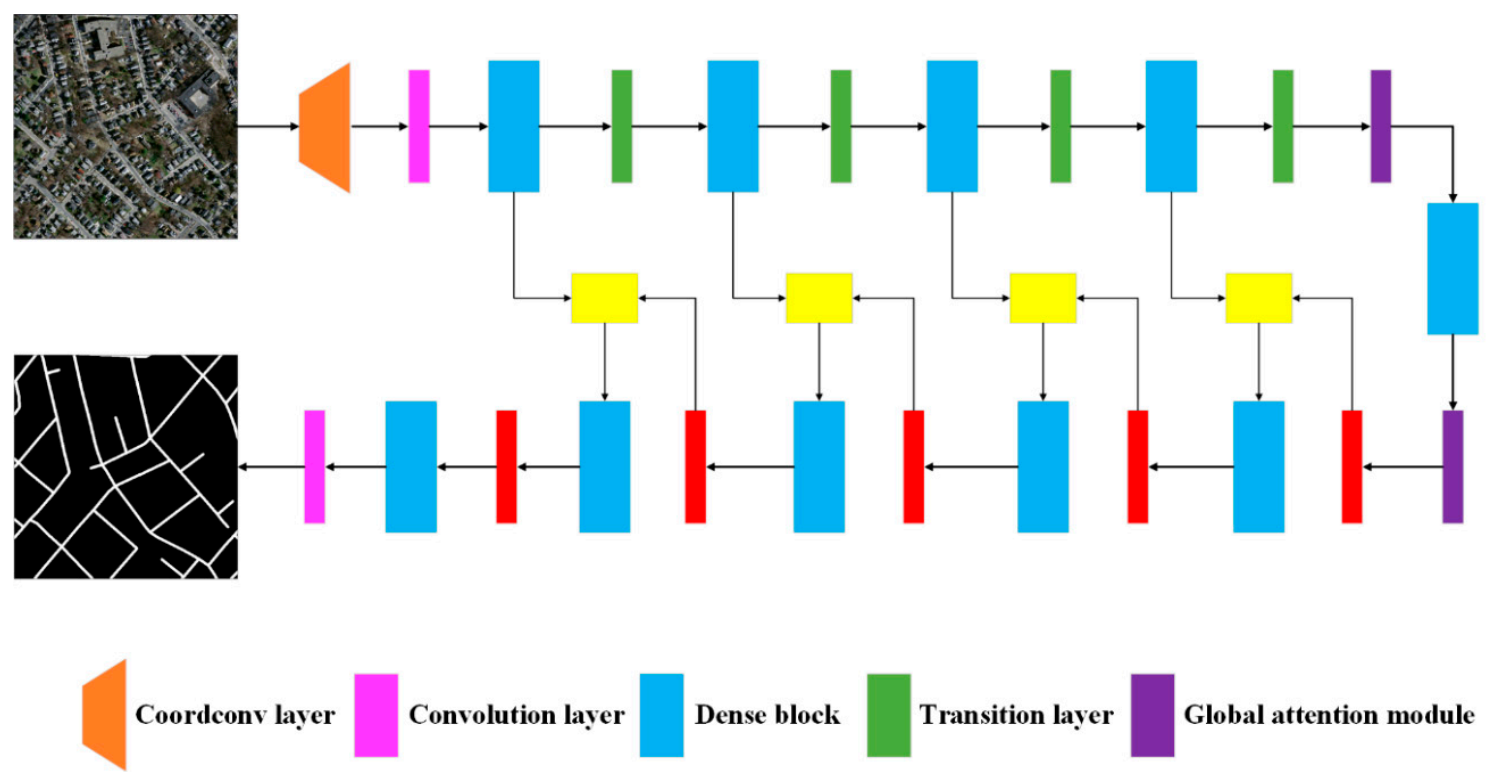

Transposed layer Skip connection

Figure 1. Structure of the proposed coord-dense-global network for improved road extraction from remote-sensing imagery.

\subsection{CoordConv Module}

The coordconv module is an extension of the standard convolution operation [34] (Figure 2a). Here, coordinate information from the original feature map is extracted and concatenated with original feature maps as input, after which standard convolution is applied (Figure 2b). In general, coordinate information is allocated in the $i$ (horizontal) and $j$ (vertical) channels. These two coordinates are sufficient to specify input pixels and improve spatial information. Another channel can be used for an $r$ coordinate, which is directly calculated from the $i$ and $j$ coordinates.

$$
r=\sqrt{(i-h / 2)^{2}+(j-w / 2)^{2}}
$$

where $h$ and $w$ are the sizes of the original feature maps.

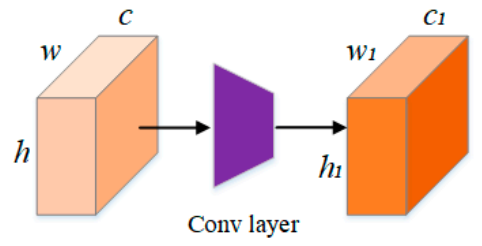

(a). Standard convolution

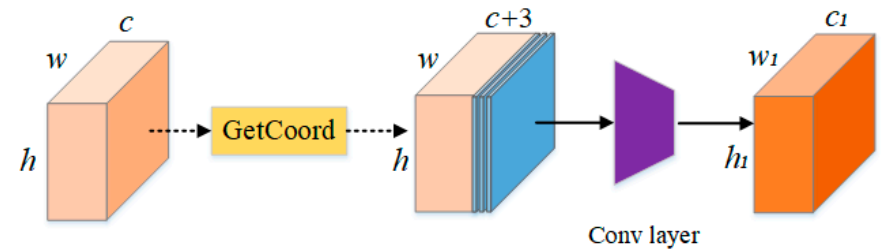

(b). CoordConv layer

Figure 2. Structural comparison between (a) a standard convolutional layer and (b) the improved module with the coordconv layer proposed in this study. 


\subsection{Global Attention Module}

As roads are thin, long, and continuous, global context information is important for extraction from remote-sensing images. Because global context information can easily enlarge the receptive field and enhance the consistency of pixelwise classification [41-43], we designed a global attention module (GAM) to strengthen high-level features for category classification (Figure 3). The GAM first applies global average pooling to the feature map, obtains the global context vector, and then performs different processing paths. On one path, the deconvolution operation is carried out first, and the results are then added to the original feature map; on the other path, the pooling operation is carried out first. After the activation, the sigmoid classifier is used to normalize the feature vector to [0,1], and then it is multiplied with the original feature map. The terminal of the module adds the output feature maps of the two paths. This module can effectively avoid the loss of global context information and strengthen high-level features while improving segmentation performance.

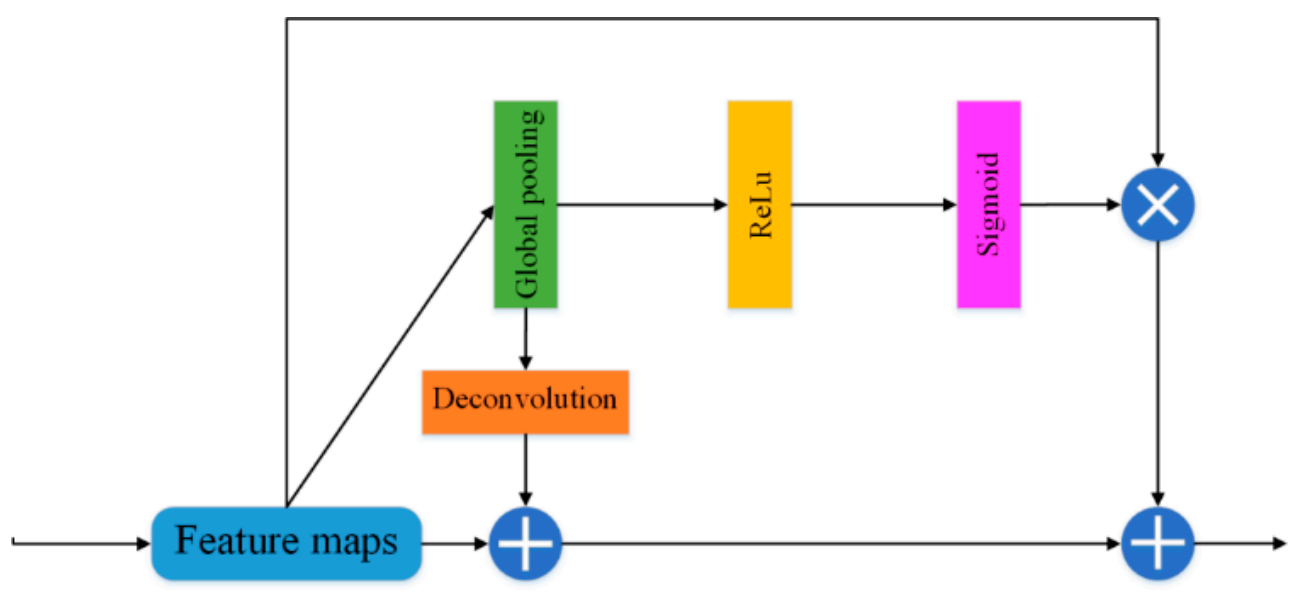

Figure 3. Structure of the global attention module proposed in this study.

\subsection{Implementation}

The concrete parameters of the proposed network are displayed in Figure 4 . The growth rate is set as 16, and the convolution operation is $3 \times 3$ with different numbers of layers in each dense block. For each transition layer, a $1 \times 1$ convolution operation and a dropout layer with a 0.2 rate are implemented, followed by a $2 \times 2$ average pooling operation. The transition layer is used to reduce the feature resolution and connect adjacent dense blocks. The transpose layers are opposite, and they recover the resolution. The transpose layer usually adopts a $3 \times 3$ convolution operation with a 2 stride, and the activation function is often a rectified linear unit (ReLu). After the end of the encoder-decoder process, the feature maps with the same resolution as the input image are output. Due to the binary classification of road extraction, the output is $512 \times 512 \times 2$. 


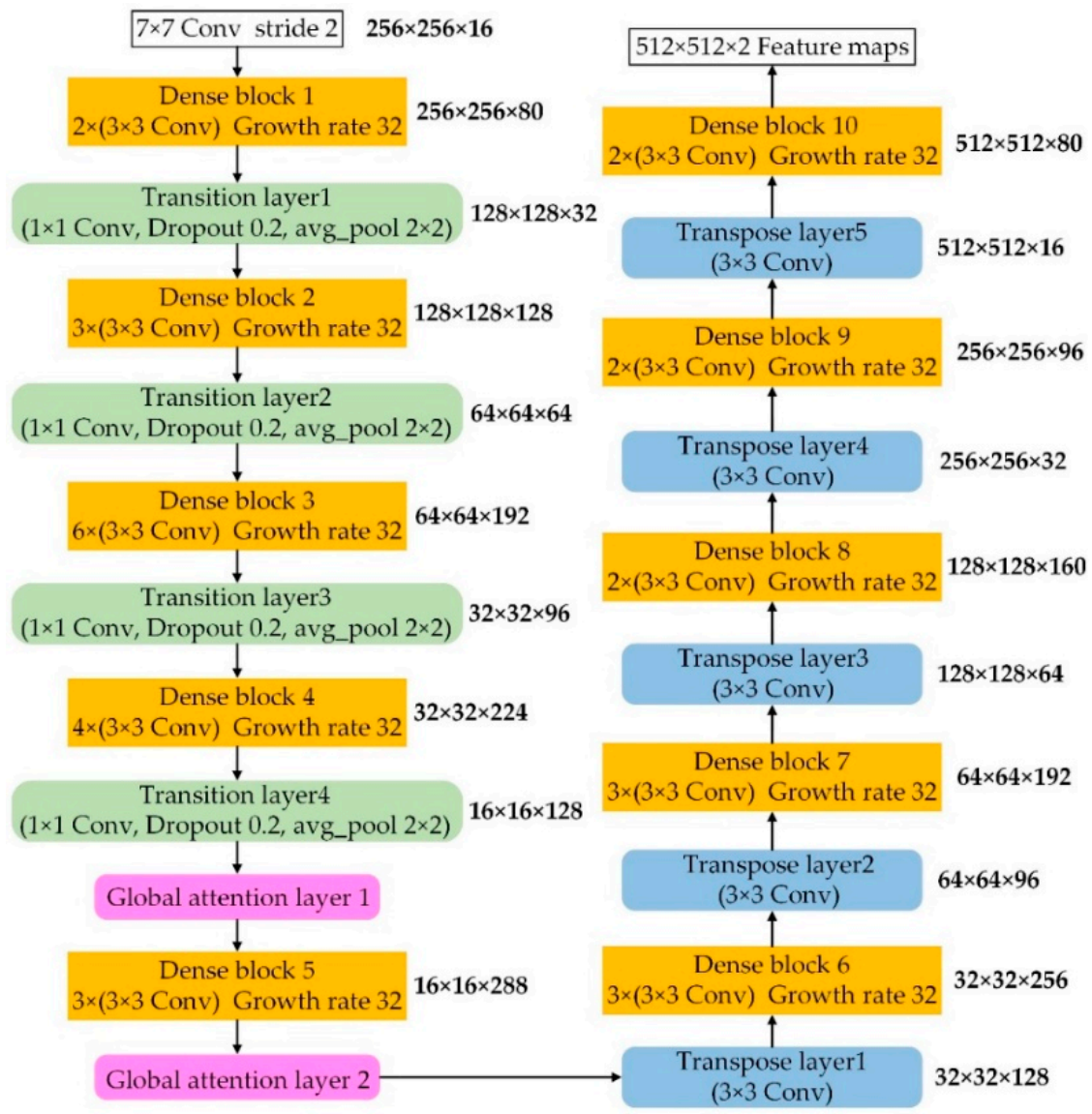

Figure 4. Detailed parameters of the coord-dense-global (CDG) model: orange boxes are dense blocks, green boxes are transition layers, purple boxes are global attention layers, and blue boxes are transition layers.

\subsection{Data}

To test the performance of the CDG model, we used a publicly available aerial imagery dataset for roads in Massachusetts, USA [44]. This dataset provided 1171 images with red, green, and blue channels, including 1108 training images, 49 test images, and 14 validation images. All images were $1500 \times 1500$ pixels with a spatial resolution of $1.2 \mathrm{~m}$. These images cover a wide range of contexts, including urban, suburban, and rural regions, covering an area of $2600 \mathrm{~km}^{2}$, and are considered a challenging aerial image labeling dataset [22].

\subsection{Implementation}

We cropped all images to $512 \times 512$ pixels. Due to the limited number of training samples, we randomly split all labeled images, producing 14,366 subset images for training. We also directly tested the testing data at $1500 \times 1500$ pixels without other processing. The training used 50 epochs and a batch size of two, according to the graphics processing unit (GPU) memory. To train the network more appropriately, we used the training epochs to automatically adjust the learning rate by dividing the training epochs into several levels and then dividing the learning rate by 10 when the epoch reached the corresponding level. The initial learning rate was 0.001 , and the final rate was 0.000001 . The total training time was approximately $50 \mathrm{~h}$, and the average testing time of each image at $1500 \times 1500$ pixels was approximately $1 \mathrm{~s}$. In addition, we used the Adam optimizer [45] to optimize our model and update all parameters because of Adam's high computational efficiency and low memory requirement. 


\subsection{Evaluation Metrics}

We used the F1 score and IoU metrics to evaluate the network's quantitative performance. The F1 score is a powerful evaluation metric for the harmonic mean of the precision and recall metrics, and it is directly calculated as:

$$
\begin{gathered}
F 1=2 \times \frac{\text { precision } \times \text { recall }}{\text { precision }+ \text { recall }} \\
\text { recall }=\frac{T P}{T P+F N}, \text { precision }=\frac{T P}{T P+F P} .
\end{gathered}
$$

where $T P, F P$, and $F N$ represent the number of true positives, false positives, and false negatives, respectively. The recall metric represents the number of correct pixels over the ground truth, while the precision metric represents the number of correct pixels over the prediction result. These values can be calculated using the pixel-based confusion matrix for each batch.

The IoU metric represents the intersection of the prediction and ground truth regions over their union, and the mean $I o U$ can be calculated by averaging the $I o U$ of all classes:

$$
I o U=\frac{T P}{F N+T P+F P}
$$

\section{Experimental Results}

After training CDG, we evaluated 49 test images, and we saw that the predicted road performance obtained by this method was good, more complete than other networks, and closer to the ground truth image. In order to accurately evaluate the performance, the average of the four evaluation indexes, the precision, recall, F1 and IoU of all test images are listed in Table 1, which are $81.41 \%, 71.80 \%, 76.10 \%$ and $61.90 \%$, respectively. At the same time, in order to intuitively understand the extraction effect of the CDG model, we show the best and worst results in the test pictures, as shown in Figure 5.

Table 1. Statistical accuracy assessments for the testing images using CDG.

\begin{tabular}{ccccc}
\hline Testing Dataset & precision & recall & F1 & IoU \\
\hline All images-average & $81.41 \%$ & $71.80 \%$ & $76.10 \%$ & $61.90 \%$ \\
\hline
\end{tabular}

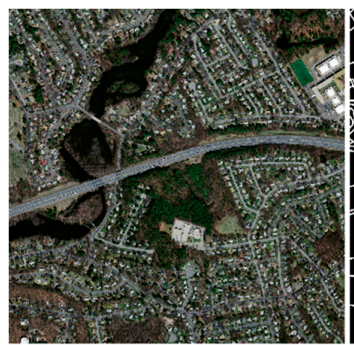

Image (a)

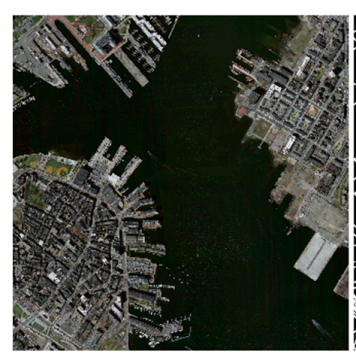

Image (d)

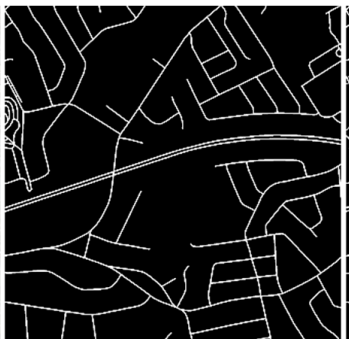

Ground truth (b)

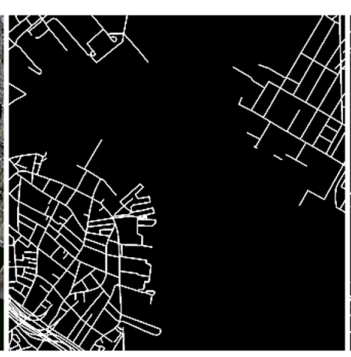

Ground truth (e)

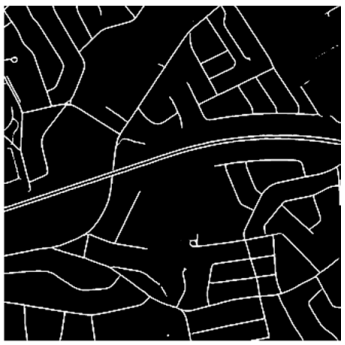

Prediction (c)

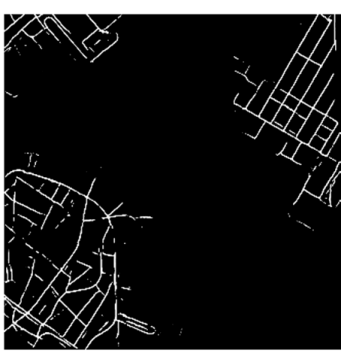

Prediction (f)

Figure 5. Comparison of the original image $(\mathbf{a}, \mathbf{d})$, ground-truth data $(\mathbf{b}, \mathbf{e})$, and extraction result $(\mathbf{c}, \mathbf{f})$ for the best $(\mathbf{a}-\mathbf{c})$ and worst $(\mathbf{d}-\mathbf{f})$ test cases for CDG. 


\section{Comparison Results and Analysis}

\subsection{Comparison with Other Methods}

To assess the relative performance of CDG, we performed road extraction on selected images using three other common methods (DeepLabV3+ [33], D-LinkNet [23], and U-net [31]) and compared the results with our own, as shown in Table 2. As seen from the table, the CDG model is clearly more accurate. Although the CDG model is $2.63 \%$ lower than the U-net model with the highest accuracy in the precision index, the CDG model has the highest $F 1$ and mean IoU evaluation index, which are $0.95 \%$ and $1.19 \%$ higher than those of D-LinkNet, respectively. Compared to U-net, they increased by $0.86 \%$ and $0.96 \%$, respectively, and far exceeded DeepLabV3+. In addition, in Table 2, we also calculated the test time of each model. Although our test time is higher than Unet, it has certain advantages in test time compared with other models. From a qualitative perspective, we can see that the extraction performance of the method in this paper has improved significantly. Figure 6 shows the road extraction details of the CDG model and the other three models.

Table 2. Mean accuracy comparisons for four road extraction methods.

\begin{tabular}{cccccc}
\hline Method & precision & recall & F1 & IoU & test time \\
\hline DeepLabV3+ & $79.16 \%$ & $60.22 \%$ & $67.64 \%$ & $51.95 \%$ & $245 \mathrm{~s}$ \\
D-LinkNet & $79.45 \%$ & $71.96 \%$ & $75.15 \%$ & $60.71 \%$ & $206 \mathrm{~s}$ \\
U-net & $84.04 \%$ & $68.90 \%$ & $75.24 \%$ & $60.94 \%$ & $167 \mathrm{~s}$ \\
\hline CDG & $81.41 \%$ & $71.80 \%$ & $76.10 \%$ & $61.90 \%$ & $196 \mathrm{~s}$ \\
\hline
\end{tabular}

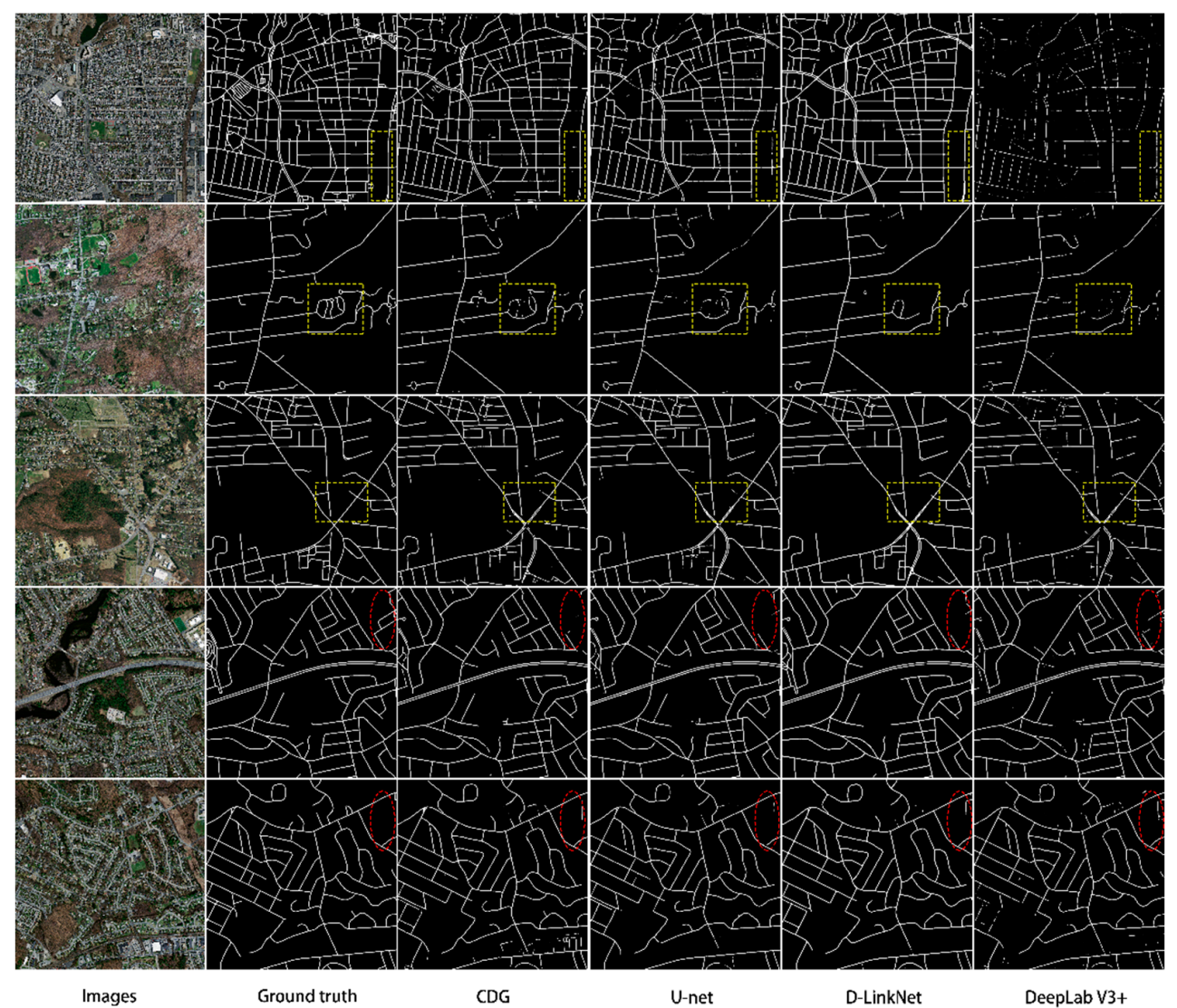

Figure 6. Comparison of results for selected images using CDG, U-net, D-LinkNet, and DeepLabV3+. Yellow boxes highlight areas with differing results between models, while red ellipses highlight areas that consistently had problems in all models. 
From the yellow box in Figure 6, we can clearly see that the extraction results of D-LinkNet and U-net are relatively fragmented, and many small roads in the remote-sensing image also have extraction losses. Among all the extraction results, DeepLabV3+ has the worst extraction effect, and the continuity and integrity of the road are difficult to guarantee. In addition, our analysis found that because the cement structure and road surface are too similar, all the methods have some misclassification problems in this case (see the red ellipses in Figure 6). However, in terms of overall performance, the extraction performance of the CDG model is still optimal.

Figure 7 is a partially enlarged image of the extraction result, which primarily reflects the ability of the CDG model to solve the problem of tree occlusion. It can be seen from the red box in Figure 7 that the CDG model performs better in extracting roads covered by trees and can extract most of the roads covered by trees, while the other three network models perform worse in extracting such roads. Although the D-LinkNet and DeepLabV3+ networks have some ability to extract occluded roads, it is difficult to ensure the continuity of the road, and the result of occluded road extraction is lost. U-net performed the worst in the problem of tree occlusion, and the degree of fragmentation and loss of extraction results was the worst. By comparing and analyzing the three network models DeepLabV3+, D-LinkNet and U-net, it can be found that the CDG network model based on dense links effectively extracts the effective feature information of the road, which improves the integrity and accuracy of the road extraction results. Regarding the coordconv module and the global attention mechanism module, the former can enhance the road edge information and make the road more complete, while the latter can consider global context information and make the road network more systematic in the extraction process. Therefore, the method of this paper can effectively counteract the interference of trees, buildings and other background features and improve the accuracy of road extraction.

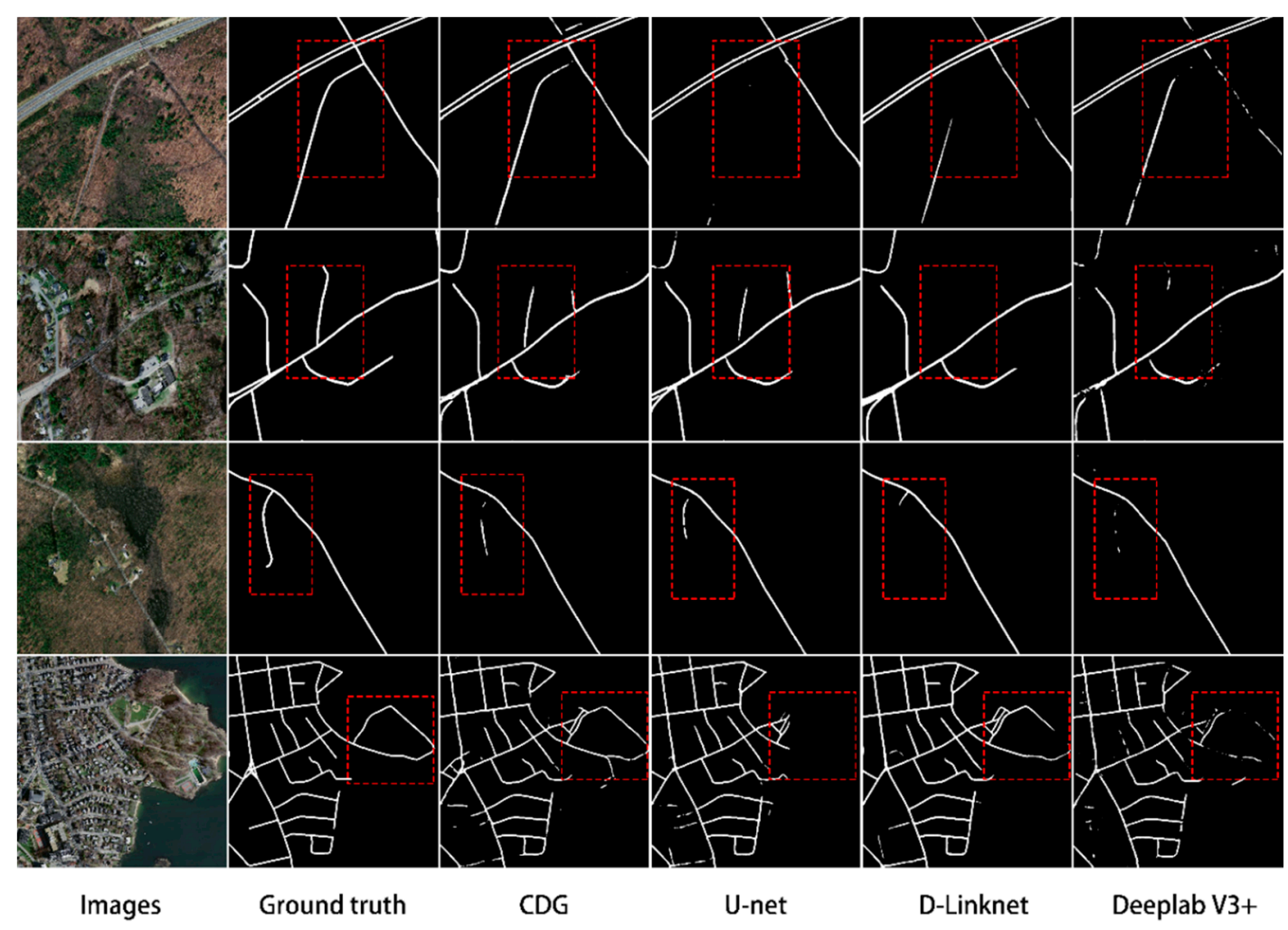

Figure 7. Comparison of results for selected images using CDG, U-net, D-LinkNet, and DeepLabV3+. Red boxes highlight areas with differing results between models. 


\subsection{Analysis of the Effectiveness of the Mechanism of Action}

To highlight the importance of coordinate convolution and the global attention mechanism, this paper compares them with network models lacking coordconv, lacking global attention and lacking both under the same training conditions. In the experiment, we also used 49 test images from the Massachusetts dataset to test the performance of different network models. To quantify the performance of the model, we list the average valuation indicators of all models in Table 3, from which we can see that the CDG model with two mechanisms is far higher than the other three methods in all evaluation indexes. Compared with the no-global-attention model with the highest accuracy, our precision, recall, $F 1$ and IoU are increased by $2.59 \%, 2.95 \%, 2.83 \%$ and $4.64 \%$, respectively. It can be seen that the combination of coordinate convolution and the global attention mechanism in the CDG model can effectively reduce the loss of road spatial features, on the one hand, and take into account the global information of the road, on the other hand; it can also enhance the integrity of the road and improve the accuracy of road extraction on the whole.

Table 3. Mean accuracy comparisons for four road extraction methods.

\begin{tabular}{ccccc}
\hline Method & precision & recall & F1 & IoU \\
\hline no coordconv & $76.12 \%$ & $60.25 \%$ & $65.75 \%$ & $49.81 \%$ \\
no global attention & $76.20 \%$ & $75.30 \%$ & $75.33 \%$ & $60.96 \%$ \\
neither & $81.40 \%$ & $57.61 \%$ & $66.76 \%$ & $50.85 \%$ \\
\hline CDG & $81.63 \%$ & $72.07 \%$ & $75.94 \%$ & $61.61 \%$ \\
\hline
\end{tabular}

\subsection{Generalization Results of the Model}

The CDG network model we designed achieved excellent results on the road data set in Massachusetts, USA. To further verify the generalization of the CDG model, we trained and tested it on the Gaofen-2 satellite remote-sensing image. The image resolution is $1 \mathrm{~m}$. In the experiment, we produced 5892 pieces of sample data with a size of $512 \times 512$. After training, we verified 4 remote-sensing images of size $4578 \times 4442$ in Hefei, China and Tianjin, China.

The test results are shown in Figure 8, we can see that the roads in the Gaofen-2 satellite remote-sensing image were extracted. Although there are missing roads in the image (see the yellow boxes in Figure 8), most of the roads in the image have been extracted; the extraction effect on the main road is particularly excellent, and the degree of agreement with the label is high. At the same time, the CDG model also extracts small roads that are not marked by the label (see the red boxes in Figure 8). To quantitatively evaluate the performance of the CDG model on the Gaofen-2 satellite remote-sensing image, we list various evaluation indicators in Table 4 . As seen from the table, the CDG model reached $68.38 \%, 77.72 \%, 72.62 \%$, and $57.11 \%$ in precision, recall, $F 1$ and $I o U$, respectively. This shows that the CDG model is has excellent extraction performance and strong generalization for road extraction tasks.

Table 4. Gaofen-2 satellite image extraction accuracy.

\begin{tabular}{ccccc}
\hline Num. & precision & recall & F1 & IoU \\
\hline 1 & $62.12 \%$ & $77.73 \%$ & $69.00 \%$ & $52.73 \%$ \\
2 & $70.88 \%$ & $75.38 \%$ & $72.54 \%$ & $56.97 \%$ \\
3 & $71.02 \%$ & $76.93 \%$ & $73.81 \%$ & $58.55 \%$ \\
4 & $69.49 \%$ & $81.83 \%$ & $75.11 \%$ & $60.20 \%$ \\
\hline Average Value & $68.38 \%$ & $77.72 \%$ & $72.62 \%$ & $57.11 \%$ \\
\hline
\end{tabular}




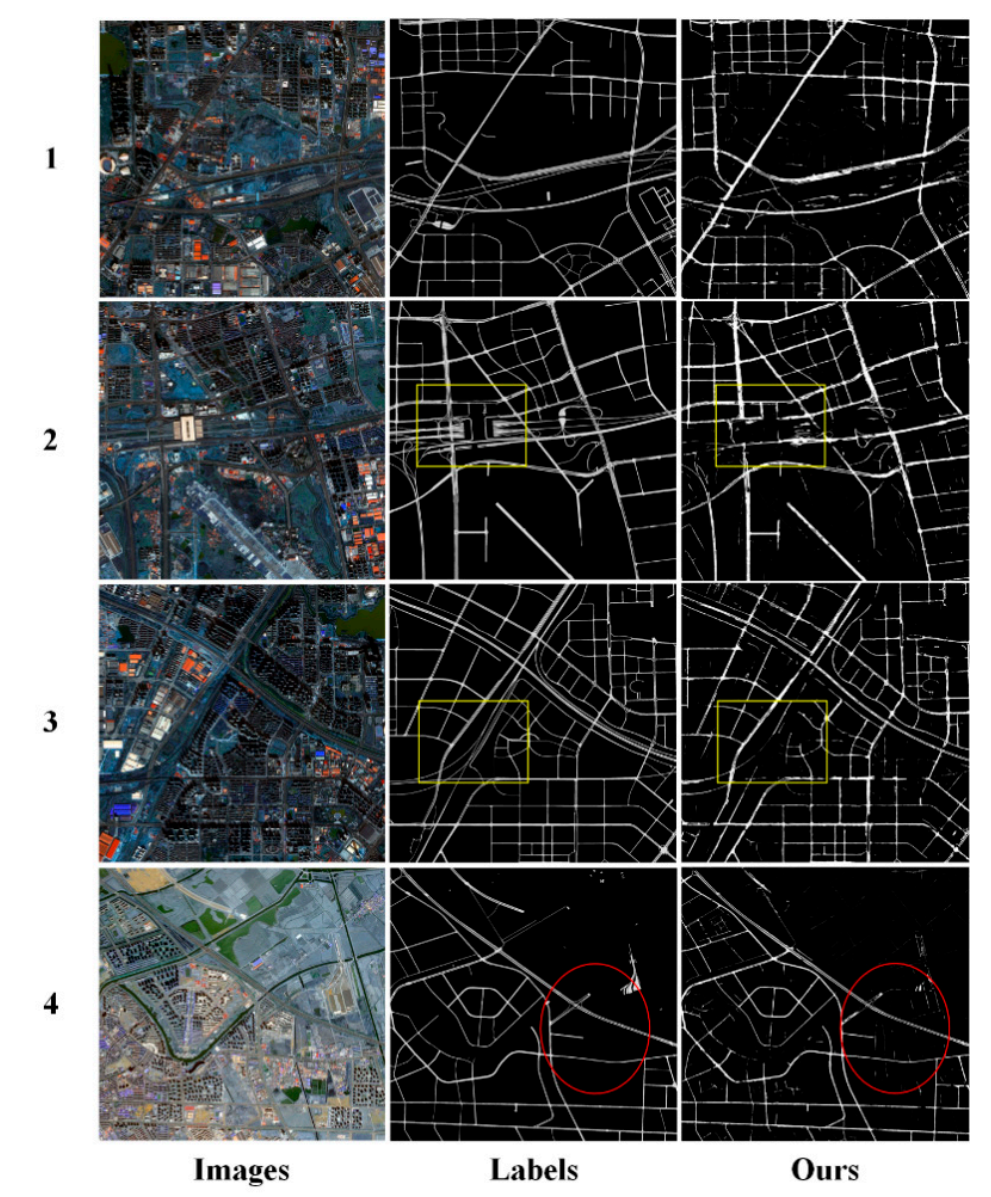

Figure 8. The results of CDG extraction on the Gaofen-2 satellite remote sensing image. Red marker box highlights excellent extraction results, while yellow ellipses highlight areas that consistently had problems.

\subsection{Problem}

Although the CDG achieved improved results, the road is extracted when the road is partially blocked by a low number of trees but, when severely blocked by many trees, the extraction effect of the CDG model is relatively poor. For example, in the previous line of Figure 9, the roads in the ground truth image are continuous (yellow boxes), but discontinuities appear in the predicted image. It may be that the roads are located in rural suburbs, and the trees above the roads are too lush, resulting in low accuracy of the extraction results; many portions of these roads are nearly indistinguishable by eye in the original image. In comparison, the lower row of Figure 9 demonstrates the superiority of the proposed model, where several roads (purple ellipses) were not labelled as roads in the ground-truth image but were successfully identified by the model. Such mismatch between ground truth images and prediction results restricts model performance, but it is difficult to further improve accuracy. 


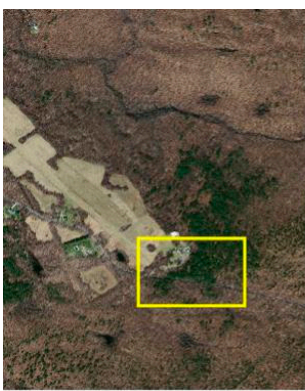

(a)

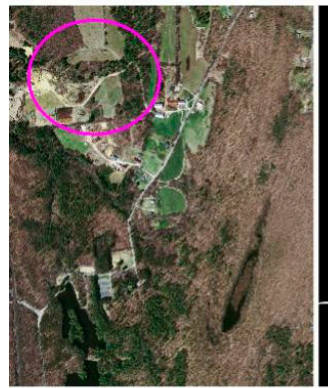

(d)

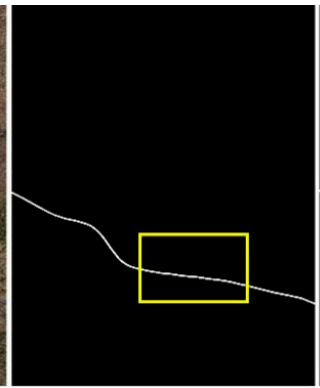

(b)

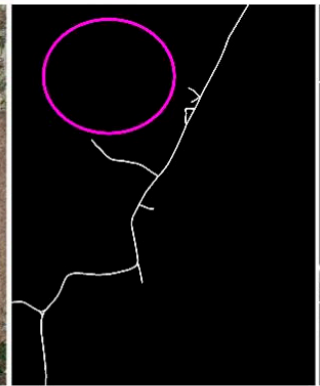

(e)

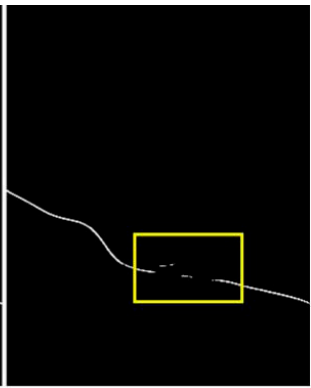

(c)

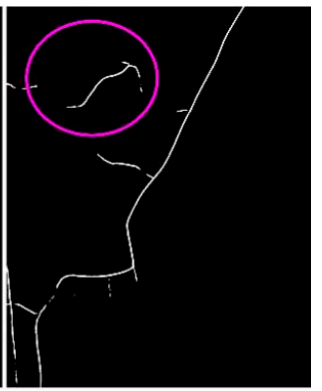

(f)

Figure 9. Two examples of problems with CDG model extraction results, comparing the (a,d) original image, $(\mathbf{b}, \mathbf{e})$ ground-truth data, and $(\mathbf{c}, \mathbf{f})$ extraction results.

\section{Conclusions}

This paper proposes a deep learning model CDG for road extraction of high-resolution remote-sensing images. The main contribution of this method lies in the introduction of two functional modules based on the Densenet network structure: coordinate convolution and ensemble attention mechanism. The cooperation of the two mechanisms can not only greatly enhance the edge information of road images, but also effectively obtain more global context information. In order to verify the performance of the model, we performed experiments on the $\mathrm{M}$ dataset and compared with the D-LinkNet, U-net, and DeepLabV3 + methods. The experiments show that the performance of the CDG model is better than other network models. At the same time, our CDG model is also the best at solving the problem of tree occlusion, and the integrity and continuity of the extraction results are significantly better than other network models. In the last universal experiment in this paper, the CDG model also successfully extracted the roads of Tianjin, China and Hefei, China, and achieved good results. Therefore, CDG is an excellent road extraction model. However, due to interference from complex backgrounds (forests, buildings, different road types and widths, etc.), the extraction results still show discontinuities. In future research, we will consider the use of road geometric information to eliminate incomplete or broken issues in the road extraction process.

Author Contributions: S.W. and Z.Z. designed the experiments, and Q.W. performed the experiments; H.Y. contributed analysis tools; S.W. wrote the paper under the guidance of Z.Z. and Y.W.; J.L. provided data processing. All authors have read and approved the manuscript.

Funding: This work was supported by the Anhui Science and Technology Major Project (grant number 18030801111), the National Natural Science Foundation of China (grant number 41971311, 41571400).

Conflicts of Interest: The authors declare no conflicts of interest.

\section{References}

1. Hinz, S.; Baumgartner, A.; Ebner, H. Modeling contextual knowledge for controlling road extraction in urban areas. In Proceedings of the IEEE/ISPRS Joint Workshop on Remote Sensing and Data Fusion over Urban Area, Rome, Italy, 8-9 November 2001; pp. 40-44. 
2. Li, Q.; Chen, L.; Li, M.; Shaw, S.L.; Nüchter, A. A sensor-fusion drivable-region and lane-detection system for autonomous vehicle navigation in challenging road scenarios. IEEE Trans. Veh. Technol. 2014, 63, 540-555. [CrossRef]

3. Bonnefon, R.; Dherete, P.; Desachy, J. Geographic information system updating using remote sensing images. Patt. Recog. Lett. 2002, 23, 1073-1083. [CrossRef]

4. Mena, J.B. State of the art on automatic road extraction for GIS update: A novel classification. Patt. Recog. Lett. 2003, 24, 3037-3058. [CrossRef]

5. Zhang, Z.; Liu, Q.; Wang, Y. Road extraction by deep residual U-Net. IEEE Geosci. Remote Sens. Lett. 2018, 15, 749-753. [CrossRef]

6. Sghaier, M.O.; Lepage, R. Road extraction from very high resolution remote sensing optical images based on texture analysis and beamlet transform. IEEE J. Sel. Top. Appl. Earth Obs. Remote Sens. 2016, 9, 1946-1958. [CrossRef]

7. Miao, Z.; Wang, B.; Shi, W.; Zhang, H. A semi-automatic method for road centerline extraction from vhr images. IEEE Geosci. Remote Sens. Lett. 2014, 11, 1856-1860. [CrossRef]

8. Shi, W.; Miao, Z.; Debayle, J. An integrated method for urban main-road centerline extraction from optical remotely sensed imagery. IEEE Trans. Geosci. Remote Sens. 2014, 52, 3359-3372. [CrossRef]

9. Kaliaperumal, V.; Shanmugam, L. Junction-aware water flow approach for urban road network extraction. IET Image Process. 2014, 10, 227-234.

10. Mu, H.; Zhang, Y.; Li, H.; Guo, Y.; Zhuang, Y. Road extraction base on Zernike algorithm on SAR image. In Proceedings of the 2016 IEEE International Geoscience and Remote Sensing Symposium (IGARSS), Beijing, China, 10-15 July 2016; pp. 1274-1277.

11. Unsalan, C.; Sirmacek, B. Road network detection using probabilistic and graph theoretical methods. IEEE Trans. Geosci. Remote Sens. 2012, 50, 4441-4453. [CrossRef]

12. Shi, W.; Zhu, C. The line segment match method for extracting road network from high-resolution satellite images. IEEE Trans. Geosci. Remote Sens. 2002, 40, 511-514.

13. Das, S.; Mirnalinee, T.T.; Varghese, K. Use of salient features for the design of a multistage framework to extract roads from high-resolution multispectral satellite images. IEEE Trans. Geosci. Remote Sens. 2011, 49, 3906-3931. [CrossRef]

14. Cheng, G.; Zhu, F.; Xiang, S.; Pan, C. Road centerline extraction via semisupervised segmentation and multidirection nonmaximum suppression. IEEE Geosci. Remote Sens. Lett. 2016, 13, 545-549. [CrossRef]

15. Senthilnath, J.; Rajeshwari, M.; Omkar, S.N. Automatic road extraction using high resolution satellite image based on texture progressive analysis and normalized cut method. J. Indian Soc. Remote Sens. 2009, 37, 351-361. [CrossRef]

16. Li, M.; Stein, A.; Bijker, W.; Zhan, Q. Region-based urban road extraction from vhr satellite images using binary partition tree. Int. J. Appl. Earth Obs. Geoinf. 2016, 44, 217-225. [CrossRef]

17. Huang, X.; Zhang, L. Road centreline extraction from high-resolution imagery based on multiscale structural features and support vector machines. Int. J. Remote Sens. 2009, 30, 1977-1987. [CrossRef]

18. Miao, Z.; Shi, W.; Zhang, H.; Wang, X. Road centerline extraction from high-resolution imagery based on shape features and multivariate adaptive regression splines. IEEE Geosci. Remote Sens. Lett. 2013, 10, 583-587. [CrossRef]

19. Wei, Y.; Wang, Z.; Xu, M. Road structure refined CNN for road extraction in aerial image. IEEE Geosci. Remote Sens. Lett. 2017, 14, 709-713. [CrossRef]

20. Geng, L.; Sun, J.; Xiao, Z.; Zhang, F.; Wu, J. Combining cnn and mrf for road detection. Comput. Electr. Eng. 2017, 70, 895-903. [CrossRef]

21. Alvarez, J.M.; Gevers, T.; Lecun, Y.; Lopez, A.M. Road scene segmentation from a single image. In Proceedings of the European Conference on Computer Vision, Florence, Italy, 7-13 October 2012.

22. Panboonyuen, T.; Jitkajornwanich, K.; Lawawirojwong, S.; Srestasathiern, P.; Vateekul, P. Road segmentation of remotely-sensed images using deep convolutional neural networks with landscape metrics and conditional random fields. Remote Sens. 2017, 9, 680. [CrossRef]

23. Zhou, L.; Zhang, C.; Wu, M. D-LinkNet: LinkNet with Pretrained Encoder and Dilated Convolution for High Resolution Satellite Imagery Road Extraction. In Proceedings of the 2018 IEEE/CVF Conference on Computer Vision and Pattern Recognition Workshops (CVPRW), Salt Lake City, UT, USA, 18-22 June 2018. 
24. Wang, Q.; Gao, J.; Yuan, Y. Embedding structured contour and location prior in siamesed fully convolutional networks for road detection. IEEE Trans. Intell. Transp. Syst. 2018, 19, 230-241. [CrossRef]

25. Buslaev, A.V.; Seferbekov, S.S.; Iglovikov, V.I.; Shvets, A.A. Fully convolutional network for automatic road extraction from satellite imagery. CVPR Workshops 2018. [CrossRef]

26. Mendes, C.C.; Frémont, V.; Wolf, D.F. Exploiting fully convolutional neural networks for fast road detection. In Proceedings of the 2016 IEEE International Conference on Robotics and Automation (ICRA), Stockholm, Sweden, 16-21 May 2016.

27. Cheng, G.; Wang, Y.; Xu, S.; Wang, H.; Xiang, S.; Pan, C. Automatic road detection and centerline extraction via cascaded end-to-end convolutional neural network. IEEE Trans. Geosci. Remote Sens. 2018, 55, 3322-3337. [CrossRef]

28. Zhong, Z.; Li, J.; Cui, W.; Jiang, H. Fully convolutional networks for building and road extraction: Preliminary results. In Proceedings of the 2016 IEEE Geoscience \& Remote Sensing Symposium, Beijing, China, 10-15 July 2016.

29. Xu, Y.; Xie, Z.; Feng, Y.; Chen, Z. Road Extraction from High-Resolution Remote Sensing Imagery Using Deep Learning. Remote Sens. 2018, 10, 1461. [CrossRef]

30. Mnih, V.; Hinton, G.E. Learning to Detect Roads in High-Resolution Aerial Images. In Proceedings of the Computer Vision-ECCV 2010-11th European Conference on Computer Vision, Heraklion, Greece, 5-11 September 2010; Proceedings, Part VI. Springer: Berlin/Heidelberg, Germany, 2010.

31. Ronneberger, O.; Fischer, P.; Brox, T. U-Net: Convolutional networks for biomedical image segmentation. In Proceedings of the Medical Image Computing and Computer-Assisted Intervention, Munich, Germany, 5-9 October 2015.

32. Badrinarayanan, V.; Kendall, A.; Cipolla, R. Segnet: A deep convolutional encoder-decoder architecture for image segmentation. arXiv 2015, arXiv:1511.00561. [CrossRef]

33. Chen, L.C.; Zhu, Y.; Papandreou, G.; Schroff, F.; Adam, H. Encoder-decoder with atrous separable convolution for semantic image segmentation. arXiv 2018, arXiv:1802.02611.

34. Rosanne, L.; Joel, L.; Piero, M.; Felipe, P.S.; Eric, F.; Alex, S.; Jason, Y. An intriguing failing of convolutional neural networks and the CoordConv solution. arXiv 2018, arXiv:1807.03247v1.

35. Yao, X.; Yang, H.; Wu, Y.; Wu, P.; Wang, B.; Zhou, X.; Wang, S. Land use classification of the deep convolutional neural network method reducing the loss of spatial features. Sensors 2019, 19, 2792. [CrossRef]

36. Gao, H.; Zhuang, L.; van der Maaten, L.; Weinberger, K.Q. Densely connected convolutional networks. arXiv 2018, arXiv:1608.06993v5.

37. Jegou, S.; Drozdzal, M.; Vazquez, D.; Romero, A.; Bengio, Y. The one hundred layers tiramisu: Fully convolutional DenseNets for semantic segmentation. arXiv 2017, arXiv:1611.09326v3.

38. Glorot, X.; Bordes, A.; Bengio, Y. Deep sparse rectifier neural networks. In Proceedings of the Fourteenth International Conference on Artificial Intelligence and Statistics, Fort Lauderdale, FL, USA, 11-13 April 2011.

39. Li, L.; Liang, J.; Weng, M.; Zhu, H. A multiple-feature reuse network to extract buildings from remote sensing imagery. Remote Sens. 2018, 10, 1350. [CrossRef]

40. Ioffe, S.; Szegedy, C. Batch normalization: Accelerating deep network training by reducing internal covariate shift. arxiv 2015, arXiv:1502.03167v3.

41. Liu, W.; Rabinovich, A.; Berg, A.C. ParseNet: Looking wider to see better. arXiv 2015, arXiv:1506.04579v2.

42. Hu, J.; Shen, L.; Sun, G.; Wu, E. Squeeze-and-excitation networks. arXiv 2018, arXiv:1709.01507v2.

43. Li, H.; Xiong, P.; An, J.; Wang, L. Pyramid attention network for semantic segmentation. arXiv 2018, arXiv:1805.10180v1.

44. Mnih, V. Machine Learning for Aerial Image Labeling. Ph.D. Thesis, University of Toronto, Toronto, ON, Canada, 2013.

45. Kingma, D.P.; Ba, J. Adam: A method for stochastic optimization. arXiv 2017, arXiv:1412.6980v9.

(C) 2020 by the authors. Licensee MDPI, Basel, Switzerland. This article is an open access article distributed under the terms and conditions of the Creative Commons Attribution (CC BY) license (http://creativecommons.org/licenses/by/4.0/). 